Boise State University

ScholarWorks

Data Management Plan for Enhancing the Humanities

Elisabeth Shook

Boise State University

Kelly Bilinski Arispe

Boise State University

Albertsons Library Research Data Management Team 


\section{Project Personnel}

Project personnel consists of the Project Director, Key Person, a Program Evaluator and undergraduate student workers for content creation, and a six-member project advisory board. Only the Project Director, Key Person, and Program Evaluator will have access to all data elements, including raw and identifiable data. Student workers will only have access to content they create for the project. Members of the advisory board will be provided reports highlighting project progress and distilled, de-identified data.

\section{EXPECTED DATA}

Data collected through this project will include: survey data collected via Qualtrics and exported as a .csv file, Zoom interviews saved as .mp4 video files, transcripts derived from Zoom interviews as .txt files, professional development workshop materials in .pdf, teacher training module materials in .pdf, analytics data in .csv to track usage of the Pathways Project website and materials on the Open Educational Resources (OER) Commons, and project reports and other resulting publications in .docx and .pdf. Tools for data cleaning and analyses will include Excel saved as .xlsx and .csv, SPSS saved as .sav, and Mplus saved as an .imp.

\section{Data Storage, Preservation, and Retention}

In compliance with Boise State University's Data Classification Standard and Boise State University Data Use Guidelines, all files will be stored in a shared Boise State Google Drive folder. Google Drive is a licensed, private instance of the Google service which provides unlimited cloud-based storage with managed back-ups. Access will be overseen by Kelly Arispe and limited to Key Person and Program Evaluator and authorized project personnel. Only the Project Director, Key Person and Program Evaluator will have access to the raw data files, which includes the Qualtrics survey data, the recorded Zoom interview videos, interview transcripts, and the analytics data.

Undergraduate student workers creating content for the Pathways Project will have access to a folder within the PI's Google Drive (separate from the raw data files) where they can create and share documentation. Similarly, any documentation and de-identified, aggregated data will be created and made available in a separate folder. When the content is ready to be shared with teachers or the advisory board, documents will be downloaded from the Google Drive as .pdf files. Copies of the .pdf files will be added to the Drive for storage and preservation. This procedure will also be followed for similar project reports or other publications resulting from the project.

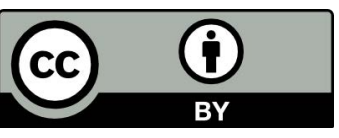


Raw data will be retained for five years, after which time the PI will evaluate the utility of keeping the identifiable survey data, video, and transcripts.

\section{Data Organization}

The data collected will be housed in one master folder titled "NEH DHAG Project, Level II". All data collection materials will be organized within the master folder, filed within subfolders labeled according to the stages outlined in the Ganntt chart in the Work Plan (1, 2 or 3) and individually named by the task of the project and shared with an editable link with the Project Director, Key Person and Program Evaluator. The ReadMe file will include a manifest and variable data and will be stored in the master folder.

Study-level documentation, created using the Boise State University Study-Level Documentation README.TXT Template, will include at a minimum: description of the data, structure of data files, and information on confidentiality, access policies, or other conditions of use, and data collection methods used. When appropriate, documentation will also include quality assurance procedures carried out, changes to data collection processes made over time, and related publications or presentations.

\section{Data Dissemination}

De-identified survey data, training materials, and reports will be shared via ScholarWorks, Boise State's Institutional Repository, at the conclusion of the project. Managed by Albertsons Library, ScholarWorks utilizes a hosted platform, optimized for open discovery. Additionally, descriptive metadata records and permanent DOIs will be created for each data set to ensure proper citation and permanent retrieval of the materials. Data sets published through ScholarWorks will be released under a modified Creative Commons license. When possible, research data will also be shared through other appropriate disciplinary repositories. 